\title{
Contribution of acid and duodenogastro- oesophageal reflux to oesophageal mucosal injury and symptoms in partial gastrectomy patients
}

\author{
M F Vaezi, J E Richter
}

\begin{abstract}
Background-The role of acid and pepsin in causing symptoms and oesophagitis is well established; however, the significance of duodenogastro-oesophageal reflux (DGOR) in this disorder is unclear.

Aims-To understand the role of acid and DGOR in causing upper gastrointestinal (GI) symptoms and oesophageal mucosal injury in partial gastrectomy (PG) patients.

Methods-Thirty two PG patients with upper GI symptoms were studied. Twenty four hour ambulatory acid and bilirubin measurements were obtained with Bilitec 2000 using glass electrode and fibreoptic sensor. Upper GI symptoms and oesophagitis were correlated with either acid or DGOR.

Results-The PG patients were a heterogeneous group: $28 \%(9 / 32)$ had mixed reflux (acid+/DGOR+); 50\% (16/32) had only DGOR (acid-/DGOR+); and 22\% (7/32) had neither (acid-/DGOR-). Upper GI symptoms were associated with both mixed reflux (69\%) and DGOR (24\%). Six patients $(67 \%)$ in the acid+/DGOR+ group had oesophagitis; no acid-/DGOR+ or acid-/DGOR- patients had oesophagitis. Mixed reflux showed a significant $(p<0.0001)$ association with oesophagitis, while DGOR did not $(\mathrm{p}=0.3)$.

Conclusions-(1) The majority of upper GI symptoms and all cases of oesophagitis in the PG patients occurred in patients who had mixed refluxate (acid and DGOR); (2) DGOR without simultaneous acid reflux may cause symptoms, but was not associated with oesophagitis in this patient group.

(Gut 1997; 41: 297-302)
\end{abstract}

Keywords: partial gastrectomy; duodenogastrooesophageal reflux; acid reflux; oesophagitis

Gastroenterology, The Cleveland Clinic Foundation, Cleveland, Ohio, USA

J E Richter

Correspondence to: Dr Joel E Richter, Chairman, Department of Gastroenterology, The Cleveland Clinic

Foundation/S40, 9500

Euclid Avenue, Cleveland, Ohio 44195, USA.

Accepted for publication 10 March 1997

Reflux of duodenal contents into the stomach is a normal physiological event occurring most commonly at night ${ }^{1}$ but also in the fasting and post-prandial daytime periods. ${ }^{2}{ }^{3}$ However, duodenogastro-oesophageal reflux (DGOR) refers to the pathological retrograde flow of duodenal contents through the pylorus into the stomach with subsequent reflux into the oesophagus. It is well established in animal and human studies that oesophageal exposure to acid and pepsin results in upper gastrointesti- nal (GI) symptoms and mucosal injury. ${ }^{45}$ Recent experimental animal studies ${ }^{6-8}$ suggest that DGOR may also cause damage to the oesophageal mucosa; however, the clinical relevance of these findings is not well established.

Partial gastrectomy patients, due to a surgically incompetent or ablated pyloric sphincter, are especially predisposed to the deleterious effects of $\mathrm{DGOR}^{9}$ and are excellent clinical models for studying the effect of prolonged oesophageal exposure to DGOR. Studies show that over $10 \%$ of patients with Billroth I and II gastrectomy have symptoms of epigastric pain, nausea, bilious vomiting, heartburn, regurgitation, and distension, but rarely have evidence of oesophageal mucosal injury. The upper GI symptoms and rare oesophagitis in this patient population have previously been attributed to DGOR based on the assumption that the surgical procedure has significantly reduced acid secretion.

The direct association of DGOR with upper GI symptoms and oesophageal mucosal injury in partial gastrectomy patients, however, has been difficult to prove due to inaccuracies in the previously available methods of DGOR detection. ${ }^{5}$ The endoscopic presence of bile in the oesophagus or stomach is a poor indicator of its role in mucosal injury. ${ }^{10}{ }^{11}$ Scintigraphic methods show conflicting results, are stationary studies, and use radiolabelled material. ${ }^{12}$ Aspiration studies of gastric and oesophageal contents for bile acid analysis have been criticised because of the short aspiration periods and limitations of the technique. ${ }^{13}$ Alternatively, $\mathrm{pH}$ monitoring has been suggested as a better technique for detecting DGOR. The assumption is that oesophageal exposure of $\mathrm{pH}>7$, also termed "alkaline" reflux, represents reflux of duodenal contents into the oesophagus ${ }^{14}$; however, $\mathrm{pH}>7$, as a measure of DGOR, is non-specific and has recently been shown to be a poor marker for DGOR. ${ }^{15-20}$

Recently, a new fibreoptic system (Bilitec 2000) was developed which detects DGOR spectrophotometrically, independent of $\mathrm{pH} .{ }^{21}$ This system utilises the optical property of bilirubin pigment in detecting bile. Bilirubin has a characteristic spectrophotometric absorption band at $450 \mathrm{~nm}$. The basic working principle of this fibreoptic system is that any absorption near this wavelength implies the presence of bilirubin and, therefore, represents DGOR. Bilitec has been validated 
independently ${ }^{22}$ and used successfully in many studies $^{18-20} 23-26$ as an objective method for assessing DGOR. Studies from Bechi's group, ${ }^{21}$ as well as our laboratory, ${ }^{22}$ show a good correlation between the Bilitec absorbance readings and bile acid concentration measurements of gastric aspirates $(r=0.71, \mathrm{p}<0.01$ and $r=0.82$, $\mathrm{p}<0.001$, respectively) using enzymatic assays. Furthermore, Bilitec detects bile acid concentrations in the range $0.01-0.10 \mathrm{mM}$, which are more representative of bile acid concentrations found in the human stomach. ${ }^{122}$

Using this new technology, $\mathrm{pH}$ monitoring, and upper GI endoscopy, we sought to characterise better the oesophageal refluxate characteristics of 32 partial gastrectomy patients and define the role of acid and DGOR in upper GI symptoms and oesophageal mucosal injury in this patient population.

\section{Materials and Methods}

The study protocol was approved by the Institutional Review Board for Human Use at the University of Alabama at Birmingham in February 1994.

\section{STUDY POPULATION}

Thirty two consecutive partial gastrectomy patients (mean age 59 years; 26 men) presenting for the evaluation of troubling upper GI symptoms were identified through the oesophageal and endoscopy laboratories of the University of Alabama at Birmingham hospitals. The subjects included 15 Billroth I and 17 Billroth II patients with a mean time since surgery for peptic ulcer disease of 27 years (range 11-45). All patients complained of moderate to severe symptoms of heartburn, abdominal pain, regurgitation, nausea, bilious vomiting, or chest pain more than twice daily necessitating medical attention. The mean duration of symptoms was 20.2 years (range 1-39), with the onset of symptoms occurring at a mean of 7.3 years (range $0.5-29$ ) after surgery.

GENERAL STUDY DESIGN

All patients had recently undergone upper gastrointestinal endoscopy (by an endoscopist blinded to the patients' symptoms). The following oesophagitis grading system was used: grade 0, normal mucosa; grade I, mucosal friability and/or oedema; grade II, erosions; and grade III, ulcerations. All subjects stopped any medication known to affect gastrointestinal motility or acid secretion for at least 48 hours before the studies. Omeprazole was discontinued one week before the study. Subjects underwent oesophageal manometry after an overnight fast to locate the lower oesophageal sphincter (LOS). After calibration, both the glass $\mathrm{pH}$ electrode and the Bilitec fibreoptic probe were passed transnasally on the same day and positioned $5 \mathrm{~cm}$ above the proximal border of the LOS. All subjects were provided with Carnation Nutran 1.5 (Clintec Nutrition Company, Deerfield, Illinois, USA) and asked to take two cans $(500 \mathrm{ml}$ total volume) for each meal. This refluxogenic liquid diet provides 2250 calories ( $16 \%$ protein, $45 \%$ carbohydrates, $39 \%$ fat), has a $\mathrm{pH}$ between 5 and 7 , and has no absorbance in the bilirubin range based on two separate 24 hour studies performed in our laboratory. Furthermore, this diet does not have food particles which may obstruct the probe tip, giving falsely high readings. ${ }^{21}$ Patients returned home with instructions to keep a diary recording symptoms, meal times, time they went to bed, and the time of rising in the morning. Patients returned the next day after 18-24 hours monitoring to have the probes removed and the diaries reviewed.

AMBULATORY OESOPHAGEAL $\mathrm{pH}$ MONITORING

The prolonged oesophageal $\mathrm{pH}$ studies were performed using a glass electrode (model lot 440 M3, Mui Scientific, Missauga, Canada) with a diameter of approximately $4.0 \mathrm{~mm}$ and a built in reference electrode $(\mathrm{Ag} / \mathrm{AgCl})$ near the end of the tip. The $\mathrm{pH}$ electrode was calibrated at $37^{\circ} \mathrm{C}$ in pH 7 and 1 buffer solutions (Fisher Scientific, Fairlawn, New Jersey, USA) before and after completing each study. The $\mathrm{pH}$ electrode was connected to a portable digital data recorder (Mark II Gold, Synectics, Irving, Texas, USA) which stored $\mathrm{pH}$ data every four seconds for up to 24 hours. After the study, the data recorded were downloaded onto an IBM compatible computer for analysis using a Gastrosoft (Gastrosoft Inc., Irving, Texas, USA) computer program. The acid $\mathrm{pH}$ data (percentage time $\mathrm{pH}<4$ ) were analysed separately for total, upright, and supine periods. Abnormal distal oesophageal 24 hour acid exposure times were defined by exceeding the 95th centile values of either one of the parameters for percentage times $\mathrm{pH}<4$ (total $>5.5 \%$, upright $>8.3 \%$, or supine $>3.0 \%$ ) obtained from previous studies in 110 healthy volunteers. ${ }^{23}$

AMBULATORY DUODENOGASTRO-OESOPHAGEAL REFLUX MONITORING SYSTEM

Prolonged oesophageal monitoring of DGOR was performed using a fibreoptic sensor, Bilitec 2000 (Synectics, Irving, Texas, USA) developed by Bechi and colleagues. ${ }^{21}$ The system consists of a miniaturised fibreoptic probe which carries light signals into the oesophagus and back to the optoelectronic system via a plastic fibreoptic bundle. The Teflon probe head has a $2.0 \mathrm{~mm}$ open groove probe across which two wavelengths of light are emitted and material sampled. Two light emitting diodes (at 470 and $565 \mathrm{~nm}$ ) represent the source for the measurement of bilirubin, the most common pigment in bile, and the reference signal (no bilirubin absorption), respectively. The portable photodiode system converts the light into an electrical signal. After amplification, the signals are processed by an integrated microcomputer, which calculates the difference of the absorbances at $470 \mathrm{~nm}$ and $565 \mathrm{~nm}$. This value is directly proportional to the bilirubin concentration in the sample under study. The period between two successive pulses from the same source (sampling time) is eight seconds. In addition, the software averages the absorbances calculated over two successive samplings in order to decrease the noise of the measurements. 


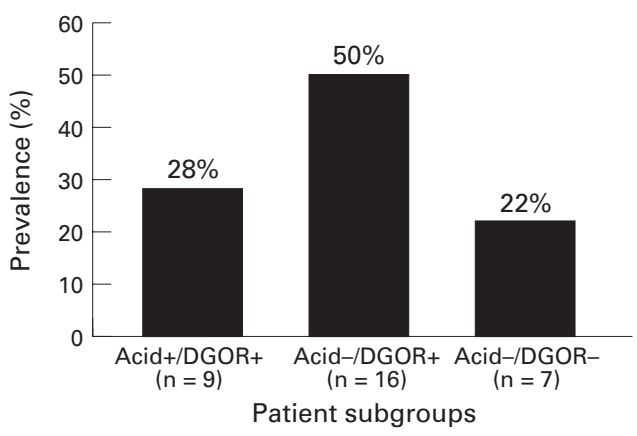

Figure 1: Prevalence of partial gastrectomy subgroups. Nine $(28 \%)$ patients had oesophageal exposure to both acid and DGOR (acid+/DGOR+), 16 (50\%) had only

DGOR (acid-/DGOR+), and the remaining seven (22\%) had neither acid nor DGOR (acid-/DGOR-).

After completion of the study, all data were downloaded onto an IBM compatible computer for analysis using the Gastrosoft computer program. The DGOR data were measured as percentage time bilirubin absorbance $\geqslant 0.14$ and analysed separately for total, upright, and supine periods. Percentage time bilirubin absorbance $\geqslant 0.14$ was chosen as values below this number represent scatter due to suspended particles and mucus present in the gastric contents. ${ }^{21}$ Abnormal 24 hour bilirubin exposure times were defined by exceeding the 95th centile values of either one of the parameters for percentage time bilirubin absorbance $\geqslant 0.14$ (total $>0.8 \%$, upright $>2.2 \%$, or supine $>1.6 \%$ ) obtained from previous studies in 20 healthy volunteers in our laboratory. ${ }^{24}$

\section{STATISTICAL ANALYSES}

All data are presented as mean (SE) and ranges of values are given where appropriate. Analysis of variance (ANOVA) was used to find the differences among partial gastrectomy subgroups in the degree of acid (percentage total time $\mathrm{pH}$

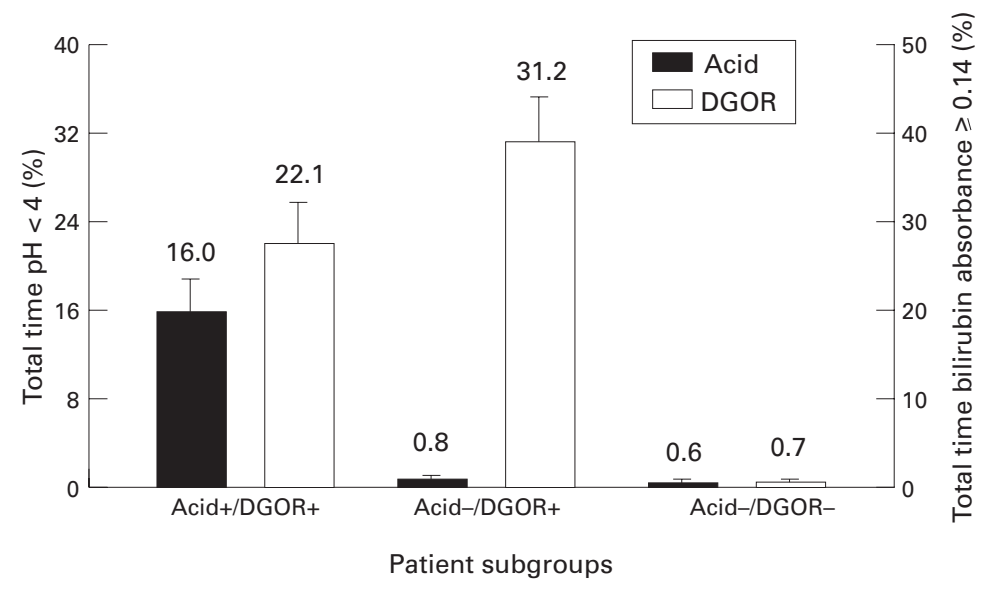

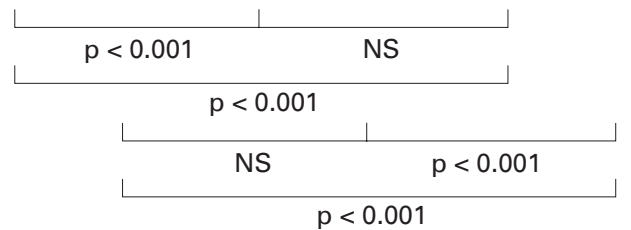

Figure 2: $\quad$ Group mean (SE) for acid (percentage total time $\mathrm{pH}<4$ ) and DGOR (percentage time bilirubin absorbance $\geqslant 0.14$ ) for the three partial gastrectomy subgroups.
$<4)$ and DGOR (percentage total time bilirubin absorbance $\geqslant 0.14$ ). The StudentNewman-Keuls multiple range test correction was used to correct for the multiple comparisons made among the partial gastrectomy subgroups. Non-parametric (Mann-Whitney) tests were performed to compare the degree of acid and DGOR among the Billroth I and Billroth II patients. The association between oesophageal mucosal injury and acid or DGOR were tested using the Fisher's exact test; $\mathrm{p}<0.05$ was considered statistically significant for all data analyses.

\section{Results}

ACID AND BILIRUBIN REFLUX

All patients completed at least 18 hours of oesophageal monitoring; there were no episodes of probe dysfunction. The mean (SE) percentage total time $\mathrm{pH}<4$ and percentage total time bilirubin absorbance $\geqslant 0.14$ for the 32 partial gastrectomy patients were 5.0 (1.9)\% (range $0.0-26.1 \%$ ) and $21.8(5.0) \%$ (range 0-91.2\%), respectively. Billroth I and II patients did not differ in their degree of acid or DGOR. The mean (SE) percentage total time $\mathrm{pH}<4$ for the Billroth I and II patients was 4.1 $(1.9) \%$ and $5.8(2.0) \%$, respectively $(p=0.3)$. The mean (SE) percentage total time bilirubin absorbance $\geqslant 0.14$ for Billroth II patients $(25.4$ $(5.4 \%))$ was higher than that for Billroth I patients (18.1 (4.5\%)); however, this difference was not statistically significant $(p=0.2)$.

Analysis of the acid and DGOR profile of the partial gastrectomy patients identified three subgroups (fig 1). Nine patients $(28 \%)$ had abnormal oesophageal exposure for both acid and DGOR (acid+/DGOR+) - that is, mixed reflux. Sixteen $(50 \%)$ had abnormal DGOR in the absence of acid reflux (acid-/DGOR+), while the remaining seven $(22 \%)$ had neither abnormal acid nor DGOR (acid-/DGOR-). Figure 2 shows the degree of acid and DGOR in each of the three partial gastrectomy subgroups. As expected, the amount of acid reflux (percentage total time $\mathrm{pH}<4$ ) in the acid+/DGOR+ group (16.0 (3.2\%)) was significantly $(\mathrm{p}<0.001)$ greater than the acid-l DGOR+ $(0.8(0.2 \%))$ or the acid-/DGOR$(0.6(0.2 \%))$ groups. The DGOR (percentage total time bilirubin absorbance $\geqslant 0.14$ ) was similar among the acid+/DGOR+ (22.1 $(4.3 \%))$ and the acid-/DGOR+ (31.2 (4.7\%)) groups, which were both significantly $(\mathrm{p}<0.001)$ higher than the acid-/DGOR- $(0.7$ $(0.2 \%)$ ) group (fig 2$)$. No patient in the study had only acid reflux. The relation between acid and bilirubin exposure times was similar for the mean percentage upright and supine times as for the previously discussed mean percentage total times.

\section{UPPER GI ENDOSCOPY}

Table 1 shows results of the upper GI endoscopy examinations in the 32 partial gastrectomy patients. Six of the 32 patients $(19 \%)$ had oesophageal mucosal injury diagnosed at endoscopy. One patient had grade I, three patients had grade II, and two patients had grade III oesophagitis. All six patients with 
TABLE 1 Prevalence of oesophagitis in partial gastrectomy subgroups

\begin{tabular}{lcl}
\hline & Oesophagitis & p Valuet \\
\hline Acid+/DGOR+ & $6 / 9(67 \%)^{\star}$ & 0.0001 \\
Acid-/DGOR+ & $0 / 16(0 \%)$ & 0.3 \\
Acid-/DGOR- & $0 / 7(0 \%)$ & 0 \\
\hline
\end{tabular}

${ }^{\star}$ Grade I, one patient; grade II, three patients; grade III, two patients.

†Association between acid/DGOR or DGOR alone with oesophageal mucosal injury (Fisher's exact test).

oesophageal mucosal injury belonged to the mixed reflux (acid+/DGOR+) subgroup (6/9 $(67 \%)$, table 1$)$. Three of the patients with mucosal injury belonged to the Billroth I group and the remaining three patients had Billroth II anastomoses. The remaining 23 patients, having a normal amount of acid reflux, had no evidence of oesophageal mucosal injury at endoscopy. There was a statistically significant $(p<0.0001)$ association between mixed reflux and oesophageal mucosal injury; DGOR did not correlate with mucosal injury $(\mathrm{p}=0.3)$.

SYMPTOM PROFILE

A total of 133 symptoms were reported by the patients during the ambulatory $\mathrm{pH} /$ bilirubin monitoring. Figure 3 summarises the individual symptoms reported and their relative contribution to the total number of symptom episodes: heartburn, 50 (38\%); abdominal pain, $40(30 \%)$; regurgitation, $18(13 \%)$; nausea/vomiting, $12(9 \%)$; and distension, 13 $(10 \%)$. Ninety two $(69 \%)$ of the 133 reported symptoms were associated with mixed reflux; $32(24 \%)$ of the symptoms were associated with DGOR.

Table 2 shows the relative contribution of the three refluxate subgroups to symptoms. The majority of symptoms of heartburn $(92 \%)$, abdominal pain $(70 \%)$, and regurgitation

TABLE 2 Percentage of upper gastrointestinal symptoms associated with the specific reflux profile

\begin{tabular}{llll}
\hline Symptoms & Acid + /DGOR+ & Acid-/DGOR+ & Acid-/DGOR- \\
\hline Heartburn & 92 & 6 & 2 \\
Abdominal pain & 70 & 30 & 0 \\
Regurgitation & 89 & 11 & 0 \\
Nausea/vomiting & 17 & 50 & 33 \\
Distension & 0 & 77 & 23 \\
\hline
\end{tabular}

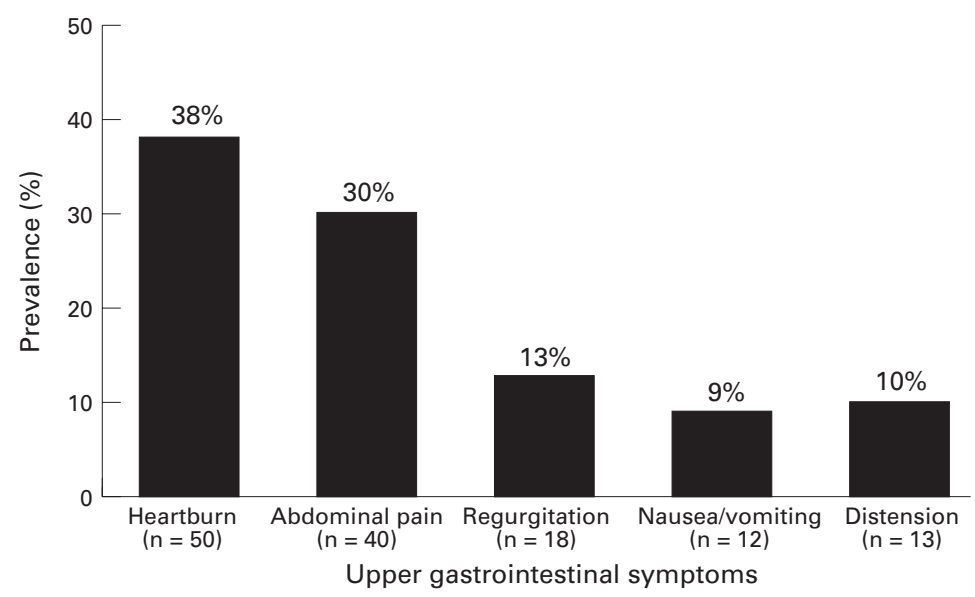

Figure 3: Prevalence of individual upper GI symptoms reported during the ambulatory $\mathrm{pH}$ and bilirubin monitoring for all the partial gastrectomy patients.
$(89 \%)$ were reported during oesophageal exposure to a mixed refluxate (acid+/ DGOR+), whereas the majority of the symptoms of nausea/vomiting ( $50 \%$ ), and distension $(77 \%)$ occurred during a non-acidic bile reflux environment (acid-/DGOR+).

\section{Discussion}

Several recent studies in patients with gastrooesophageal reflux disease (GORD) and in those with Barrett's oesophagus implicate both acid and DGOR (mixed reflux) in the development of oesophageal mucosal injury ${ }^{18-20} 25-28$; however, these studies were unable to differentiate the individual role of each constituent in causing mucosal damage. Using the new ambulatory DGOR detection technology, our study of patients after ulcer surgery provides a unique insight into the clinical importance of DGOR, alone and in combination with acid reflux, in causing oesophageal mucosal injury and upper GI symptoms.

We found that $28 \%$ of the 32 partial gastrectomy patients (fig 1) had sufficient mean acid reflux parameters (percentage total time $16.0 \%$ ) to equal oesophageal reflux parameters found among non-surgical GORD patients with oesophagitis or even patients with uncomplicated Barrett's oesophagus. ${ }^{18}{ }^{19}$ This agrees with several recent studies ${ }^{29-31}$ suggesting that acid production may not be completely eliminated in some partial gastrectomy patients. For example, $\mathrm{Machida}^{29}$ found that the decrease in gastric acidity was usually incomplete in patients undergoing emergency gastrectomy for perforated duodenal ulcer. Using gastric $\mathrm{pH}$ monitoring in patients with Billroth II anastomosis, Mann et $a l^{30}$ showed that $\mathrm{pH}$ less than 4 was present in more than $30 \%$ of a patient group with recurrent ulcer. Similarly, studies by Sears et $a l^{31}$ found that $23 \%$ of partial gastrectomy patients had reflux of acid $(\mathrm{pH}$ $<4$ ) into the distal oesophagus.

Additionally, we found that patients having undergone partial gastrectomy are a heterogeneous group of patients consisting of three subgroups: those that have mixed reflux of acid concomitant with DGOR (acid+/DGOR+, $28 \%$ ), those with only DGOR (acid-/ DGOR+, 50\%), and those that have neither acid nor DGOR (acid-/DGOR-, 22\%) (fig 1). Among the 32 partial gastrectomy patients, oesophageal mucosal injury occurred only in the subgroup of patients who had mixed refluxate (6/9 acid+/DGOR+ patients; table 1$)$. More importantly, there was no oesophagitis in the subgroup of patients with only DGOR (acid-/DGOR+; table 1), suggesting that DGOR in the absence of acid reflux may not be damaging to the human oesophagus. This is consistent with the animal experiments by Lillemoe et $a l^{\beta 2}$ which showed that taurocholate produced no oesophageal mucosal damage at a neutral $\mathrm{pH}$, but in an acidic medium $(\mathrm{pH} 1-2)$ there was oesophageal mucosal disruption as measured by net ion permeability.

Furthermore, our study confirms that the reflux of either acid/DGOR or DGOR alone can produce oesophageal symptoms (table 2). Overall, $81 \%$ of the upper GI symptoms 
reported by the partial gastrectomy patients were attributed to three symptoms: heartburn, abdominal pain, and regurgitation (fig 3), which were predominantly found in the subgroup of patients with mixed reflux (acid $+/$ DGOR+, table 2). Symptoms of nausea, vomiting, and distension, which accounted for only $19 \%$ of the overall reported symptoms (fig 3), were the predominant symptoms in the partial gastrectomy patients with non-acidic DGOR (acid-/DGOR+, table 2). Therefore, in the absence of a bilirubin monitoring device, patients' symptoms may be a useful guide for identifying the subgroups of partial gastrectomy patients who might benefit from a therapeutic trial of acid suppressive agents or promotility drugs.

The findings in our study have important implications with respect to the role of individual gastric and duodenal contents in causing symptoms and mucosal injury in humans. Our findings suggest that the contribution of non-acidic DGOR, consisting mainly of unconjugated bile acids and trypsin, ${ }^{7}$ to significant oesophageal injury in humans is minimal, while still producing upper GI symptoms. However, as suggested in the animal models, oesophageal exposure to DGOR in combination with $\mathrm{HCl}$ (mixed reflux) may result not only in upper GI symptoms, but can also cause oesophagitis. In fact, a recent study ${ }^{24}$ found that acid and DGOR occur simultaneously in about $80 \%$ of patients with GORD, suggesting a possible synergy between acid and duodenal contents in causing oesophageal mucosal injury. Perfusion studies of rabbit oesophagus show that in an acidic environment, conjugated bile acids are the main DGOR ingredients responsible for oesophageal mucosal injury. ${ }^{78}$

It is important to remember that Bilitec measures reflux of bilirubin and not bile acids; thereby presuming that the presence of bilirubin in the refluxate is accompanied by other duodenal contents. Although this is true in most cases, a few medical conditions (Gilbert's and Dubin-Johnson syndromes) may result in disproportionate secretion of bilirubin as compared with other duodenal contents, especially bile acids. Additonally, this study does not specifically address the role of pepsin in causing oesophageal mucosal injury. However, it is clear from several previous studies that pepsin plays a critical role in an acidic oesophageal refluxate. For example, using the canine oesophagus, Redo et $a^{\beta 3}$ reported no oesophageal damage with $\mathrm{HCl}$ infusion alone, while acid in combination with low concentrations of pepsin, at $\mathrm{pH}$ less than 2, caused the most severe oesophagitis. Separating the role of pepsin from acid in the production of oesophagitis is difficult, as the optimum $\mathrm{pH}$ for the enzymatic activity of pepsin is below $3 .^{34}$ However, it seems that the degree of contribution by pepsin to oesophageal mucosal injury in patients with varying severity of GORD is not related to the pepsin concentration. Recent data by Hirschowitz ${ }^{35}$ found no difference between pepsin concentration among patients with Barrett's oesophagus, oesophagitis, and healthy subjects. Furthermore, animal studies show that bile acids diminish the degree of mucosal damage caused by pepsin. For example, Lillemoe et $a b^{36}$ found that the degree of oesophageal mucosal injury and permeability decreased in a dose dependent manner when increasing concentrations of the conjugated bile acid, taurodeoxycholate $(2,5$, or $10 \mathrm{mM})$, were added to pepsin $(0.3 \mathrm{mg} / \mathrm{ml})$.

Therefore, identifying partial gastrectomy patients with abnormal amounts of mixed acid/DGOR reflux and treating them aggressively is important. Medical therapy, especially the proton pump inhibitors, notably decreases acid reflux, while simultaneously decreasing DGOR by reducing the amount of gastric volume available to reflux from the stomach. ${ }^{18}$ Furthermore, conjugated bile acids and pepsin are inactivated at the higher intragastric and intraoesophageal $\mathrm{pH}$ environments created by the proton pump inhibitors. Antireflux surgery is more problematic in partial gastrectomy patients because of their prior gastric resections and oesophageal disection. On the other hand, therapy should be directed at improving gastric emptying and preventing DGOR in the subgroup of partial gastrectomy patients with non-acidic reflux. Recent studies by Vaezi et a ${ }^{37}$ suggest that the promotility drug cisapride (20 mg four times daily) can significantly decrease DGOR by Bilitec measurements, producing symptomatic improvements in $70 \%$ of these patients. Alternatively, these patients should do well with Roux-en-Y surgical diversion.

1 Gotley DC, Morgan AP, Ball D, Owens RW, Cooper MJ. Composition of gastro-oesophageal refluxate. Gut 1991; 32: $1093-9$

2 Schidlbeck NE, Heinrich C, Stellard F, Paumgartner G, Muller-Lissner SA. Healthy controls have as much bile reflux as gastric ulcer patients. Gut 1987; 28: 1577-83.

3 King PM, Pryde A, Heading RC. Transpyloric fluid movement and antroduodenal motility in patients with gastroesophageal reflux. Gut 1987; 28: 545-8.

4 Goldberg HI, Dodds WJ, Gee S, Montgomery C, Zboralske FF. Role of acid and pepsin in acute experimental esophagitis. Gastroenterology 1969; 56: 223-30.

5 Vaezi MF, Singh S, Richter JE. Role of acid and duodenogastric reflux in esophageal mucosal injury: a review of animal and human studies. Gastroenterology 1995; 108: $1897-907$.

6 Salo JA, Kivilaakso E. Contribution of trypsin and cholate to the pathogenesis of experimental alkaline reflux esophagitis. Scand f Gastroenterol 1984; 19: 875-81.

7 Harmon JW, Johnson LF, Maydonovitch CL. Effects of acid and bile salts on the rabbit esophageal mucosa. Dig Dis Sci 1981; 26: 65-72.

8 Kivilaakso E, Fromm D, Silen W. Effect of bile salts and related compounds on isolated esophageal mucosa. Surgery 1980; 87: 280-5.

9 Brough WA, Taylor TV, Torrance HB. The surgical influencing duodenogastric reflux. Br f Surg 1984; 71: $770-3$.

10 Nasrallah SM, Johnston GS, Gadacz TR, Kim KM. The significance of gastric bile reflux seen at endoscopy. $\mathcal{F}$ Clin Gastroenterol 1987; 9: 514-7.

11 Stein HJ, Smyrk TC, DeMeester TR, Rouse J, Hinder RA. Clinical value of endoscopy and histology in the diagnosis of duodenogastric reflux disease. Surgery 1992; 112: 796-804.

12 Drane WE, Karvelis K, Johnson DA, Silverman ED. Scintigraphic evaluation of duodenogastric reflux. Problems, pitfalls, and technical review. Clin Nucl Med 1987; 12: 377-84.

13 Mittal RK, Reuben A, Whitney JO, McCallum RW. Do bile acids reflux into the esophagus? A study in normal subjects and patients with GERD. Gastroenterology 1987; 92: 371-5.

14 Pellegrini CA, DeMeester TR, Wernly JA, Johnson LF, Skinner DB. Alkaline gastroesophageal reflux. Am $\mathcal{f}$ Surg 1978; 75: 177-84.

15 Singh S, Bradley LA, Richter JE. Determinants of oesophageal "alkaline" $\mathrm{pH}$ environment in controls and patients with gastro-oesophageal reflux disease. Gut 1993; 34: 30916.

16 Devault KR, Georgeson S, Castell DO. Salivary stimulation mimics esophageal exposure to refluxed duodenal contents. Am $\mathcal{F}$ Gastroenterol 1993; 88: 1040-3. 
17 Mattioli S, Pilotti V, Felice V, Lazzzari A, Zannoli R, Bachi ML, et al. Ambulatory 24 hour pH monitoring of the 38 .

18 Champion G, Richter JE, Vaezi MF, Singh S, Alexander R Duodenogastroesophageal reflux: relationship to $\mathrm{pH}$ and importance in Barrett's esophagus. Gastroenterology 1994 107: 747-54.

19 Vaezi MF, Richter JE. Synergism of acid and duodenogastroesophageal reflux in complicated Barrett's esophagus. Surgery 1995; 117: 699-704.

20 Marshall REK, Anggiansah A, Owen WA, Owen WJ. Oesophageal acid and bile reflux correlate well: no relationship to alkaline $\mathrm{pH}$ [abstract]. Gut 1996; 38 (supp 1): A25.

21 Bechi P, Paucciani F, Baldini F, Cosi F, Falciai R, Mazzanti $\mathrm{R}$, et al. Long-term ambulatory enterogastric reflux monitoring. Validation of a new fiberoptic technique. Dig Dis Sci 1993; 38: 1297-306.

22 Vaezi MF, LaCamera RG, Richter JE. Bilitec 2000 ambulatory duodenogastric reflux monitoring system. Studies on tory duodenogastric reflux monitoring system. Studies on its validation

23 Richter JE, Bradley LA, DeMeester TR. Normal 24-hour ambulatory esophageal $\mathrm{pH}$ values. Influence of study center, pH electrode, age, and gender. Dig Dis Sci 1992; 37 849-56.

24 Vaezi MF, Richter JE. Role of acid and bile in gastroesophageal reflux disease. Gastroenterology 1996; 111: 1192-9.

25 Kauer WK, Burdiles P, Ireland AP, Clark GW. Does duodenal juice reflux into the esophagus of patiens with complicated GERD? Evaluation of a fiberoptic sensor for bilirubin. Am f Surg 1995; 169: 98-104.

26 Kauer WK, Peters JH, DeMeester TR, Ireland AP, Bremner CG, Hagen JA, et al. Mixed reflux of gastric and duodenal juice is more harmful to the esophagus than gastric juice alone. Ann Surg 1995; 222: 525-33.
27 MacKay CK, Caldwell MTP, Stuart RC, Walsh TN, Baxter JN, Byrne PJ, et al. Barrett's oesophagus: the role of oesophageal bile reflux [abstract]. Gut 1996; 38 (suppl 1):

28 Marshall RFK, Anggiansah A, Owen WA, Owen WJ. Duodenal contents in the oesophagus: are they a major cause of reflux symptoms [abstract]? Gut 1996; 38 (suppl 1): A41.

29 Machida T. A study of intragastric $\mathrm{pH}$ in patients with peptic ulcer with special reference to the clinical significance of basal pH values. Gastroenterologia faponica 1981; 16: 447-58.

30 Mann O, Glaser J, Pausch J, Rosemeyer D. Prognostic value of long-term pH-metry in the B-II resected stomach. $Z$ of long-term pH-metry in the
Gastroenterol 1993; 31: 392-4.

31 Sears RJ, Champion G, Richter JE. Characteristics of partial gastrectomy (PG) patients with esophageal symptoms of duodenogastric reflux. Am f Gastroenterol 1995; 90: 211-5.

32 Lillemoe KD, Johnson LF, Harmon JW. Role of the components of the gastroduodenal contents in experimental acid esophagitis. Surgery 1982; 92: 276-84.

33 Redo SF, Barnes WA, de la Sierra AO. Perfusion of the canine esophagus with secretions of the upper gastrointestinal tract. Ann Surg 1959; 149: 556-64.

34 Lillemoe KD, Johnson LE, Harmon JW. Alkaline esophagitis: a comparison of the ability of components of gastroduodenal contents to injure rabbit mucosa. Gastroenterology 1983; 85: 621-8.

35 Hirschowitz BI. Critical analysis with appropriate controls, of gastric acid and pepsin secretions in clinical esophagitis. Gastroenterology 1991; 101: 1149.

36 Lillemoe KD, Johnson LF, Harmon JW. Taurodeoxycholate modulates the effects of pepsin and trypsin in experimental esophagitis. Surgery 1985; 97: 662-7.

37 Vaezi MF, Sears R, Richter JE. Placebo-controlled trial of cisapride in postgastrectomy patients with duodenogastoesophageal reflux. Dig Dis Sci 1995; 41: 754-63. 